\title{
Genetic approaches to human renal agenesis/hypoplasia and dysplasia
}

\author{
Simone Sanna-Cherchi • Gianluca Caridi • \\ Patricia L. Weng • Francesco Scolari • \\ Francesco Perfumo • Ali G. Gharavi • \\ Gian Marco Ghiggeri
}

Published online: 3 July 2007

(C) IPNA 2007

\section{Erratum to: Pediatr Nephrol}

DOI 10.1007/s00467-007-0479-1

Table 2 shows some of the principal human malformation syndromes with kidney hypoplasia/dysplasia. The branchio-oto-renal syndrome is caused by mutations of EYA1, SIX1 or SIX5 genes and not SIX2. We apologize for the mistake in the article.

The online version of the original article can be found at http://dx.doi. org/10.1007/s00467-007-0479-1.

S. Sanna-Cherchi $\cdot$ P. L. Weng · A. G. Gharavi

Department of Medicine, Division of Nephrology,

Columbia University College of Physicians and Surgeons,

New York, NY, USA

S. Sanna-Cherchi

Department of Clinical Medicine,

Nephrology and Health Science, University of Parma,

Parma, Italy

G. Caridi $\cdot$ G. M. Ghiggeri $(\bowtie)$

Laboratory of Pathophysiology of Uremia, Istituto G. Gaslini,

Largo G. Gaslini 5,

16148 Genoa, Italy

e-mail: labnefro@ospedale-gaslini.ge.it

P. L. Weng

Department of Pediatrics, Division of Nephrology,

Mount Sinai School of Medicine,

New York, NY, USA

F. Scolari

Division and Chair of Nephrology, Spedali Civili,

University of Brescia,

Brescia, Italy

F. Perfumo

Division of Nephrology, Istituto G. Gaslini,

Genoa, Italy 
Table 2 List of human malformation syndromes with kidney hypoplasia/dysplasia ( $M C D K$ multicystic dysplastic kidney, VUR vesicoureteral reflux)

\begin{tabular}{|c|c|c|c|}
\hline Gene & Human syndrome & Kidney phenotype & OMIM \\
\hline JAG1, NOTCH2 & Alagille syndrome & MCDK, kidney dysplasia, kidney mesangiolipidosis & \#118450, \#610205 \\
\hline BBS1-BBS11 & Bardet-Biedl syndrome & Renal dysplasia and calyceal malformations & \#209900 \\
\hline EYA1, SIX1, SIX5 & Branchio-oto-renal syndrome & Renal agenesis/dysplasia & \#113650 \\
\hline SOX9 & Campomelic dysplasia & Diverse renal malformations & \#114290 \\
\hline CHD7 & CHARGE syndrome & Diverse urinary tract malformations & \#214800 \\
\hline Del. 22q11 & Di George syndrome & Renal agenesis, dysplasia, VUR & \#188400 \\
\hline GATA3 & $\begin{array}{l}\text { Hypothyroidism, sensorial deafness, } \\
\text { renal anomalies (HDR) }\end{array}$ & Renal agenesis, dysplasia, VUR & \#146255 \\
\hline DNA repair & Fanconi anemia & Renal agenesis & \#227650 \\
\hline FRAS1, FREM2 & Fraser syndrome & Renal agenesis, dysplasia & \#219000 \\
\hline KALL1, FGFR1 & Kallman's syndrome & Renal agenesis, dysplasia & \#308700, \#147950 \\
\hline PAX2 & Renal coloboma syndrome & Renal hypoplasia, MCDK, VUR & \#120330 \\
\hline TCF2 & Renal cysts and diabetes syndrome & Renal dysplasia, cysts & $\# 137920$ \\
\hline GPC3 & Simpsom-Golabi-Behmel syndrome & Renal dysplasia, cysts & \#300209 \\
\hline DHCR7 & Smith-Lemli-Opitz Syndrome & Renal dysplasia, cysts & \#270400 \\
\hline SALL1 & Townes-Brocks Syndrome & Renal dysplasia, lower urinary tract malformations & \#107480 \\
\hline LMX1B & Nail-patella syndrome & Glomerulus malformation, renal agenesis & \#161200 \\
\hline NIPBL & Cornelia de Lange syndrome & Renal dysplasia & $\# 122470$ \\
\hline CREBBP & Rubinstein-Taybi syndrome & Renal agenesis & \#180849 \\
\hline WNT4 & Rokitansky syndrome & Renal agenesis & \#277000 \\
\hline PEX-family & Zellweger syndrome & Renal dysplasia, cysts & \#214100 \\
\hline GLI3 & Pallister-Hall syndrome & Renal agenesis, dysplasia & $\# 146510$ \\
\hline p57(KIP2) & Beckwith-Wiedemann syndrome & Renal dysplasia & \#130650 \\
\hline SALL4 & Okihiro syndrome & $\begin{array}{l}\text { Renal ectopia with or without fusion, lower } \\
\text { urinary tract malformations }\end{array}$ & \#607323 \\
\hline TBX3 & Ulnar-mammary syndrome & Renal agenesis & $\# 181450$ \\
\hline
\end{tabular}

